\title{
Demonstration of an Accumulation of Galactose-1- Phosphate in the Liver from Congenital Galactosemia
}

\author{
By \\ Keiya Tada \\ From the Department of Pediatrics, Faculty of Medicine, Tohoku. \\ University, Sendai; Director: Prof. Ts. Arakawa \\ (Received for publication, July 19, 1962)
}

Congenital galactosemia is an inborn error of metabolism characterized by an inability to metabolize galactose. It has been established ${ }^{1-3)}$ that the disease is due to congenital absence or deficiency in the enzyme, galactose-1-phosphate uridyl transferase, which catalyzes the conversion of galactose-1-phosphate (Gal1-P) to glucose-1-phosphate. This enzyme defect may lead to an accumulation of Gal-1-P, which may in turn cause cellular injury. Schwarz et al. $\left.{ }^{4}\right)$ found the accumulation of large amounts of Gal-1-P in the erythrocytes of galactosemic patients after they were given milk or after their erythrocytes were incubated with galactose in vitro. However, the direct evidence of accumulation of Gal-1$P$ in the liver of the patient with this particular disorder is, as far as we know, lacking despite that the liver is the major organ for galactose metabolism.

In this paper, chromatographical demonstration of Ga-1-P in the liver of a patient with congenital galactosemia is reported.

Case. A 4-year-old girl was brought to our Clinic because of retardation of physical and mental development. Physical examinations revealed a poorly $\mathrm{n}$ urished child with a yellow tinge over the skin. The abdomen was distended, but there was no fluctuation. The liver was firm in consistence and palpable 3 fingerbreadths, and the spleen 3 fingerbreadths below the costal margin. $\mathrm{X}$-ray films of the bones exhibited findings of osteoporosis and rickets. The intelligence was remarkably retarded, indicating 52 of I.Q. Laboratory findings revealed a striking galactosuria and a typically galactosemic curve in the galactose tolerance test. The case was thus diagnosed as congenital galactosemia. This diagnosis was further confirmed by Schwarz's method ${ }^{5}$ : - The erythrocytes from the patient was incubated with galactose, then an abnormal accumulation of Gal-1-P in the erythrocytes was demonstrated paperchromatographically (cf. Fig. 1). 


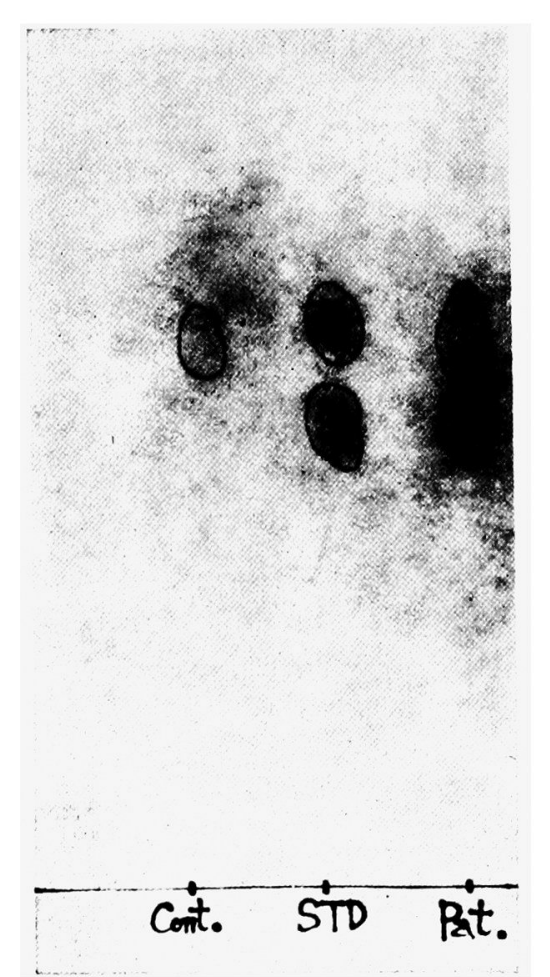

Fig. 1. Schwarz's test

STD: Standard solution; the upper spot indicates glucose-phosphate and the lower spot galactose-phosphate.

Cont: Control subject, showing no detectable spot of galactose-phosphace.

Pat: Galactosemic patient, showing a remarkable spot of galactose-phosphate.

In order to demonstrate the accumulation of Gal-1-P in the liver, a liver specimen was taken from the patient by exploratory laparotomy. At the laparotomy, the liver was found to be of uneven surface and firm in consistence. Histological findings of the liver showed a picture of the Laennec type of liver cirrhosis (cf. Fig. 2). The liver specimen obtained at this laparotomy was utilized for identification of a possible accumulation of Gal-1-P in the liver.

\section{METHODS}

The isolation and identification of hexose-phosphate from the hepatic tissue were carried out after the method which Schwarz et al. employed in the case of erythrocytes. The liver specimen, after being washed sufficiently with saline solution, was homogenized with a chilled isotonic KCL solution to make a $2 \%$ homogenate. To $2 \mathrm{ml}$ of the homogenate, $6 \mathrm{ml}$ of ice-cold $20 \%$ trichloracetic acid were added and the mixture was kept in iced water for ten minutes with occasional 


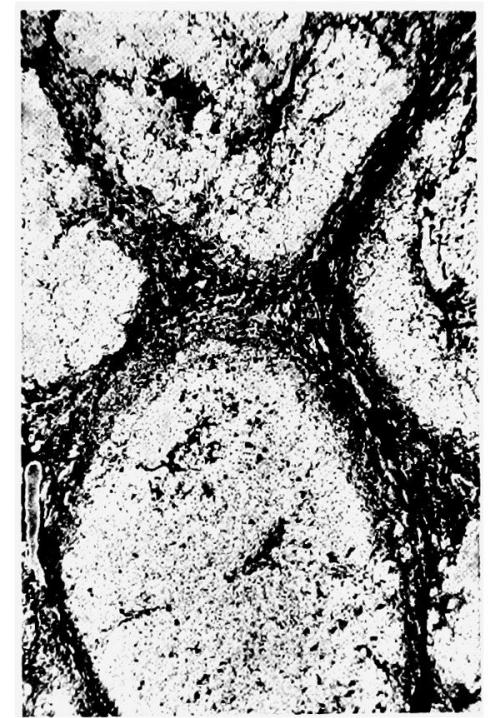

Fig. 2. Pathological finding of the liver biopsied from the patient with congenital galactosemia.

Mallory's stain, a picture of liver cirrhosis (Laennec type).

shaking. After centrifuging, the supernant was filtered. To the filtrate, 2 drops of $25 \%$ barium acetate were added, then the mixture was made slightly alkaline (phenolphthalein being used as the indicator) by the addition of $40 \% \mathrm{NaOH}$. The phosphates were precipitated by the addition of 4 volumes of ethanol and by being kept overnight in the refrigerator. Then the precipitate was spun down, the supernant removed, and the precipitate washed once with $5 \mathrm{ml}$ of neutralized ethanol. After an addition of $1.5 \mathrm{ml}$ of water, the precipitate was broken up with a glass rod, and the mixture was neutralized with $1 \mathrm{~N}$ HCL and then made up to the acidity of $0.1 \mathrm{~N}$ by the addition of $1 \mathrm{~N}$ HCL. The hexose-phosphate was hydrolysed by keeping the mixture in boiling water for 7 minutes. After cooling, about $0.5 \mathrm{ml}$ of water was added, followed by a slight excess of $1 \mathrm{~N}_{2} \mathrm{SO}_{4}$ to precipitate the barium. The mixture was neutralized with $2 \mathrm{~N} \mathrm{NH}_{4} \mathrm{OH}$ and centrifuged. The supernant fluid was transferred to a small evaporating-cup and was evaporated to dryness in a vacuum desiccator. The dried residue was dissolved in $0.5 \mathrm{ml}$ of pyridine by grinding it with a small glass rod.

The solids were sedimented by sharp centrifugation for 4 or 5 minutes. $0.2 \mathrm{ml}$ of the pyridine extract was spotted on a paperchromatogram. For the help of identification, a standard solution containing glucose and galactose was spotted alongside the sample. The chromatogram was run overnight in butanol/ pyridine/water (30/20/15 by volume). After drying the chromatogram, a freshly prepared aniline reagent $-930 \mathrm{mg}$ of aniline and $1.6 \mathrm{~g}$ of phthalic acid 
in $100 \mathrm{ml}$ of the water-saturated-n-butanol - was sprayed on the chromatogram. The chromatogram was heated for 5 minutes in an oven at $100^{\circ} \mathrm{C}$ for developing color spots for each of hexose.

As a control, a liver specimen was obtained by surgical operation from a patient without any liver involvement. The control specimen also was treated for chromatography in the same way as the specimen from the galactosemic patient.

\section{RESULTS}

A distinct spot of galactose, which was thought to be derived from Gal-1-P, was found in the case of the galactosemic patient, while there was no detectable spot of galictose in the case of the control subject (cf. Fig. 3). The liver specimen was taken from a galactosemic patient by exploratory laparotomy after about 18 hour's fasting except for being given glucose solution. Therefore, this evidence the detection of galiatose-1-phosphate in the liver of the patient - will show that Gal-1-P accumulates in the liver as the result of a block on way of galactose metabolism, i.e. as the result of a defect of Gal-1-P uridyl transferase.

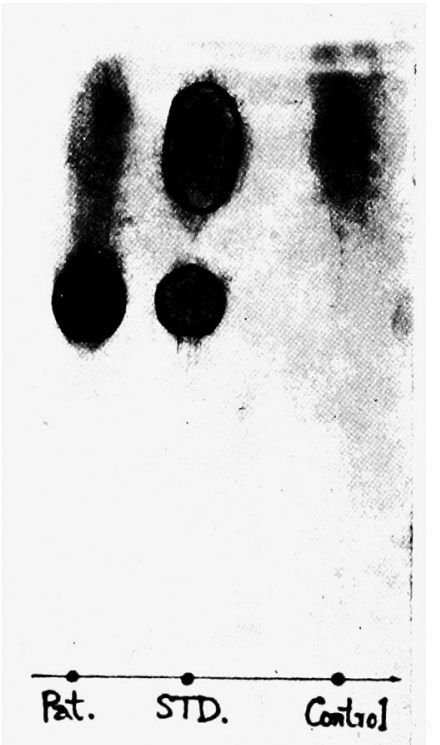

Fig. 3. Demonstration of galactose-phosphate in the liver of the patient with congenital galactosemia.

STD: Standard solution; the upper spot indicates glucose-phospate and the lower spot galactose-phosphate.

Cont: Control subject, showing no detectable spot of galactosephosphate.

Pat: Galactosemic patient, showing a remarkable spot of galactosephosphate. 


\section{DISCUSSION}

Formerly it had been supposed by many authors ${ }^{8-12)}$ that galactose, found in a high level in the blood or tissues, might be the noxious agent in case of congenital galactosemia. However, since the demonstration of a defect of Gal-1-P uridyl transferase in the liver and erythrocytes of galactosemic patients by Kalckar and his co-workers ${ }^{1-3)}$, a new hypothesis ${ }^{13,14}$ ) has appeared that the toxic agent is not galactose itself but galactose-1-phosphate which may accumulate in the tissues of galactosemic patients. The most convincing evidence for this new hypothesis was obtained through the study of the growth characteristics of K-12 E. Coli galactose negative mutants by Kurahashi and Wahba. ${ }^{15}$ ) They found that the addition of galactose to the growth medium of E. Coli lacking galactokinase resulted in no alteration in the rate of growth, whereas there was, as they saw, a significant retardation of growth when galactose was added to the medium of Gal-1-P uridyl transferase-less mutants. They ${ }^{15)}$ also showed an accumulation of Gal-1-P in those uridyl transferase-less mutants grown in a medium containing galactose, whereas there was, as they further showed, no increase in Gal-1-P within the cells of the galactokinase-less mutants grown in a similar medium.

Schwartz et al. demonstrated an accumulation of Gal-1-P in the erythrocytes of galactosemic patients when fed galactose $\left.{ }^{4}\right)$ and in the lens of rats fed on a high galactose or lactose diet. ${ }^{16)}$ Kosterlitz $^{17}$ ) found that Gal-1-P accumulated in the livers of rabbits fed on a high galactose diet.

In this paper, we could demonstrate an accumulation of Gal-1-P in the liver of a patient with congenital galactosemia. The liver is the major organ for galactose metabolism and is most severely affected in case of congenital galactosemia, resulting in occurrence of jaundice and finally in liver cirrhosis. The present evidence - a demonstration of Gal-1-P in the liver of galactosemic patient - suggests that the accumulation of Gal-1-P may be responsible for the development of liver cirrhosis in this paritcular disorder through the injurious effect of Gal-1-P for the cellular metabolism. ${ }^{18,19)}$

\section{SUMMARY}

In the liver of a patient with congenital galactosemia an accumulation of galactose-1-phosphate was demonstrated by paperchromatography.

\section{References}

1) Kalckar, H.M. et al., Proc. Acad. Sc., 1956, 42, 49.

2) Isselbacher, K.J. et al., Science, 1956, 123, 635.

3) Anderson, E.P. et al., Science, 1957, 125, 113.

4) Schwarz, V. et al., Biochem. J., 1956, 62, 34.

5) Schwarz, V. et al., Lancet, 1958, 1, 24.

6) Mellinkoff, S. et al., J. Pediatrics, 1945, 27, 338.

7) Townsend, E.H. et al., Pediatrics, 1951, 7, 760. 
8) Bruck, E. \& Rapaport, S., Am. J. Dis. Child., 1945, 70, 267.

9) Cusworth, D.C. et al., Arch. Dis. Child., 1955, 30, 150.

10) Hsia, D.Y. et al., Am. J. Dis. Child., 1954, 88, 458.

11) Bray, P.T. et al., Arch. Dis. Child., 1952, 27, 341.

12) Johns, D., Am. J. Dis. Child., 1953, 85, 575.

13) Holzel, A. et al., Am. J. Med., 1957, 22, 703.

14) Kirman, H.N., Metabolism, 1960, 9, 316.

15) Kurahashi, K. \& Wahba, A.J., Biochim. et Biophys. Acta, 1958, 30, 298.

16) Schwarz, V. \& Goldberg, L., ibid. 1955, 18, 310.

17) Kosterlitz, H.W., Biochem. J., 1943, 37, 318.

18) Tada, K. et al., Tohoku J. Exp. Med., 1962, 77, 400.

19) Gardner, L.T., Molecular Genetics and Human Disease, 1961, 61, Charles C Thomas Publisher, Springfield, Illinois. 